\title{
Construction of Mental Health Education System in Higher Vocational Colleges Based on Web Platform
}

\author{
$\mathrm{YanHu}^{1}$ \\ ${ }^{1}$ Chongqing college of architecture and technology, 401331
}

\begin{abstract}
This article combines the principles of constructing a health system based on the Web platform to discuss the relevant content of the Web platform technology. The principles of constructing a health system based on the Web platform include the principle of teaching students in accordance with their aptitude, the principle of taking students as the main body, the principle of god effectiveness in achieving goals, and the principle of initiative task. The author studied the system construction elements such as overall system design, functional module design, database module design, interactive platform module design, and resource sharing platform design. The purpose of this article is to improve the application effect of the health system based on the Web platform and improve the mental health of students in higher vocational colleges.
\end{abstract}

\section{Introduction}

Mental health education is an important part of college education. It is not only the support of quality education, but also an important guarantee for the harmony and stability of universities. Good mental health education can not only promote the benign development of college students' mental health, but also contribute to their psychological growth. At the same time, it has laid a good foundation for students' all-round development and adaptation to society. Therefore, colleges and universities need to carry out mental health education effectively and use the advantages of Web platform applications to build a mental health education system in higher vocational colleges. This can not only improve the teaching effect of mental health courses, but also guide students' ideological development correctly.

\section{Overview of Web Platform Technology}

In web design, web means web page. Now the web is widely translated into network and internet. In specific applications, it is mainly divided into the following three application methods. (1) Hypertext. This is a global information structure that links different parts of the document through keywords, so that information can be searched interactively. (2) Hypermedia. Hypermedia is a combination of hypertext and multimedia in an information browsing environment. Not only can users jump from one text to another, but they can also activate a sound, display a graphic, and even play an animation. If users want to know the content of a certain topic, they only need to click on the topic to jump to the document that contains this topic. This provides users with application convenience. (3) Hypertext Transfer Protocol (HTTP). It refers to the transmission protocol of hypertext on the
Internet.

\section{Principles of Constructing Mental Health System Based on Web Platform}

\subsection{Individualized Teaching Principle}

Judging from the current application situation, more than $90 \%$ of the mental health education in higher vocational colleges is carried out in a collective form. However, this teaching mode has certain inherent defects in its application. For example, there are certain differences in the psychological quality of students themselves, and there are also different ways of thinking. Therefore, the use of "equalization" teaching methods is not conducive to the improvement of all students' comprehensive ability. In the process of building a mental health system, we need to consider the differences in students' own abilities and clarify the differences in students' learning. Afterwards, according to the relevant situation of the difference, reasonably arrange the course content. This is conducive to the advancement of the mental health of all students in the right direction, thereby improving the practicality of course content education.

\subsection{Student-centered Principle}

More than $70 \%$ of higher vocational colleges still have teachers as the main body in the teaching of mental health courses. Students are relatively passive when learning in the classroom, which is not conducive to the improvement of classroom teaching effects. Moreover, the mental health curriculum is to cultivate students' personal abilities. Therefore, teachers should change their traditional concepts, regard students as the main body of teaching, 
and carry out corresponding teaching activities on this basis. This can effectively optimize the entire teaching process, encourage students to actively participate in the course of learning, and use this to improve the effect of course teaching [1].

\subsection{Goal Achievement Principle}

Mental health courses are similar to other higher vocational courses. In specific applications, they need to achieve the three-dimensional goals shown in Figure 1. They are the improvement of students' knowledge and skills, the improvement of emotional attitudes and values, the mastery of processes and methods. In the process of building the system, we also need to meet the principle of effectiveness, so that it can meet the requirements of classroom development, and then show the results of the system.

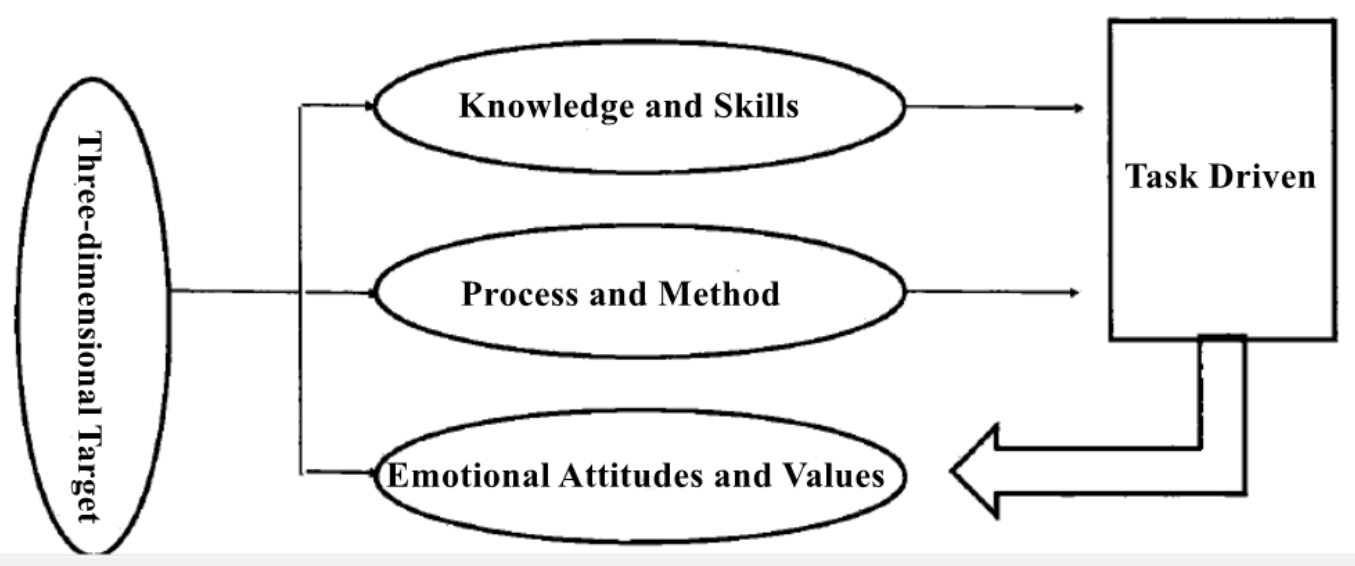

Fig1. Schematic Diagram of Three-dimensional Target

\subsection{InitiativeTaskPrinciple}

As mentioned above, because the mental health curriculum is to cultivate students' personal abilities, teachers should change their traditional concepts. The construction of a mental health system should follow the principle of task initiative and use various methods to cultivate students' learning initiative. For example, some small tasks with difficulty ranging from simple to difficult can be set, so that students can complete the study work of the course according to the predetermined requirements. In addition, in this process, the corresponding application rules can also be improved, thereby improving the quality of task completion and improving students' classroom learning effects [2].

\section{Essentials of Constructing Mental Health Education System in Higher Vocational Colleges Based on Web Platform}

\subsection{Overall System Design}

\subsubsection{Student Module Design}

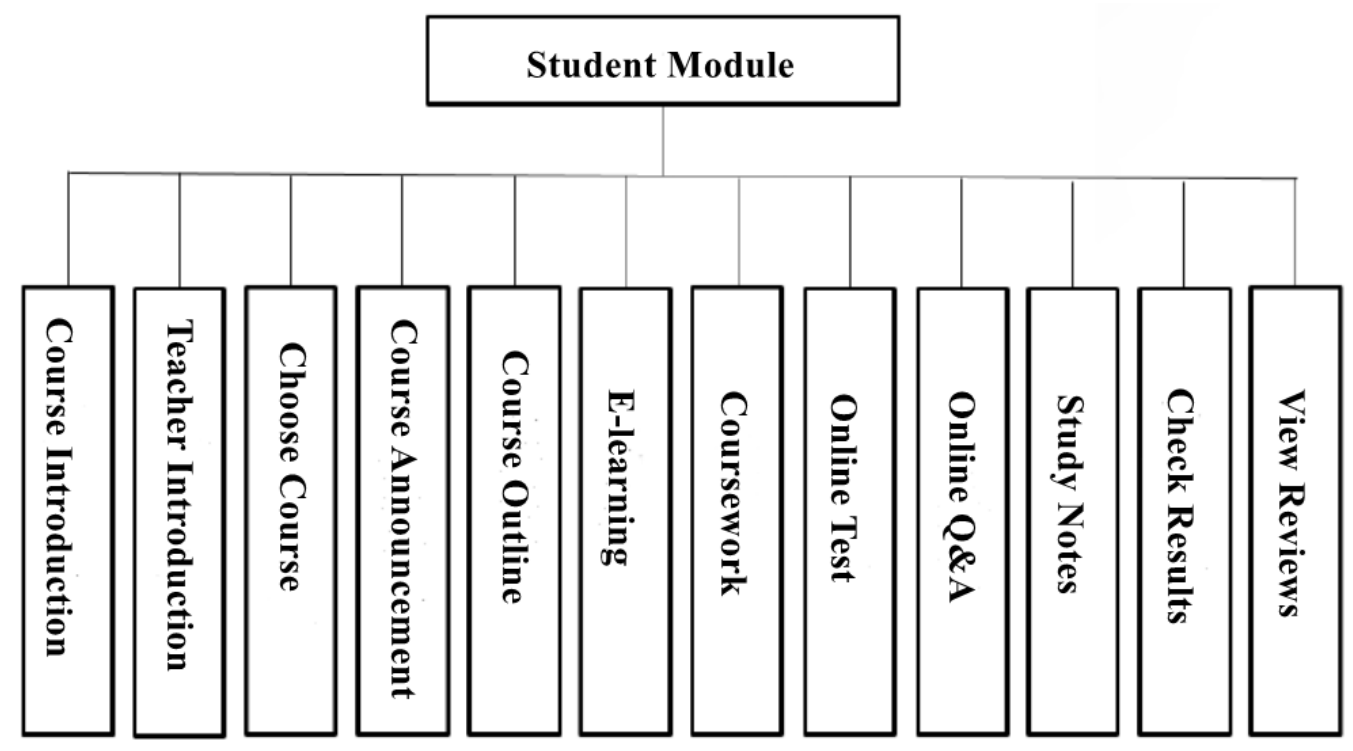

Fig2. Schematic Diagram of the Student Module 
Students are the main body of course learning, and student module design is a very important application link in the overall design. After logging in to the Web platform, students can basically understand the course content and teacher information, and then select the required course content. Students can choose to watch online or download courseware [3]. After completing the course, students need to submit coursework online. At the same time, teachers also need to complete online corrections. In this way, students can get feedback from the teacher in time and ask questions about teaching. Afterwards, teachers regularly conduct online answers to form a systematic teaching process.

\subsubsection{Teacher Module Design}

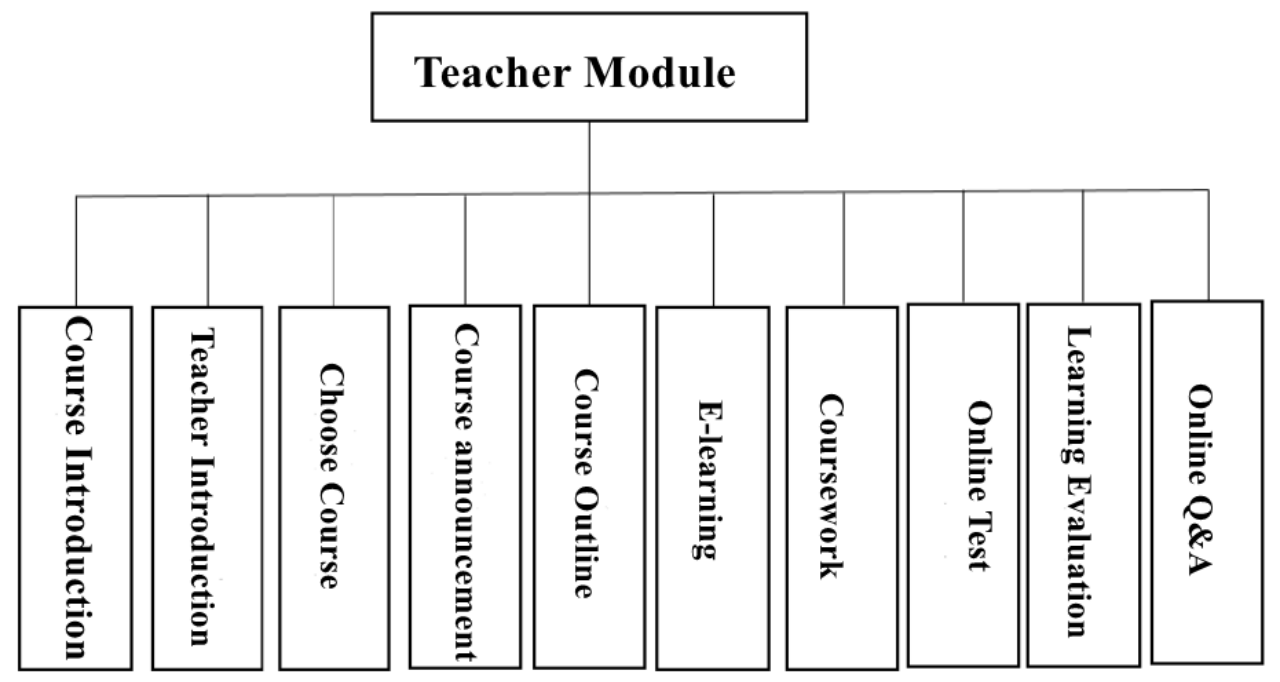

Fig3. Schematic Diagram of Teacher Module Classification

As shown in Figure 3, after logging in to the Web platform, teachers can learn about the content of the course selected by the student, and then conduct online teaching based on the content of the course selected by the student. Teachers sometimes choose the method of recording courseware to provide students with reference materials. Besides, the teacher will reserve course assignments after the course teaching ends, and students are required to complete and submit the assignments within the specified time. Teachers complete the online correction work according to the requirements, and grade the students' homework [4]. Students can use the platform to get feedback in time and raise teaching questions to teachers. Teachers answer specific questions based on the content of questions raised by students. In addition, teachers can also record student questions and add them to follow-up instructional videos to help students better learn course knowledge.

\subsubsection{Administrator Module Design}

In addition to designing the student and teacher modules, we also need to design the administrator module. The administrator module coordinates and manages all content in the Web platform. In specific applications, it is mainly registered by system administrators themselves. Its task is to perform system update, BUG repair, personnel or course cleaning and other tasks. With the graduation of vocational college students, the login accounts of these students also need to be cancelled. As a result, the administrator needs to clean it up, and the information of the resigned teachers will also be deleted to ensure the reliability of the platform login environment [5]. Moreover, managers are also responsible for the assignment of permissions. They can issue different application permissions for students and teachers, thereby enhancing the reliability of the content of the education system.

\subsection{Functional Module Design}

\subsubsection{System Login Module}

In the study of mental health courses, teachers need to focus on improving students' comprehensive ability. According to the different foundations and abilities of different students, various types of application courses are provided in the platform to facilitate students to complete the required courses [6]. Among them, the user login module is one of the very important basic modules. When users $\log$ in, they need to $\log$ in with a user name and password, and the back-end system will determine the identity of the loginer to jump to different selection pages, thereby improving the pertinence and rationality of the selected content.

\subsubsection{Course Learning Module}

After logging in to the Web platform, students can start to select the required course content through the course learning module. In the design process of this module, we can refer to the e-mail interaction method and rely on the application advantages of the Web platform to display the course content such as basic mental health knowledge and mental health videos. Furthermore, we can also divide modules on the interface according to information type functions. For example, we can set it as an unread message section, a course information section, a learning progress section, a reminder section for submitting homework, a 
course list and other modules [7]. Meanwhile, in order to optimize the content of the system, we can also build a similar information interaction interface to improve the application effect of the course learning module.

\subsubsection{Information Query Module}

This module is mainly designed for the management of administrators. According to the needs of education reform, the mental health education knowledge that was not applicable in the past is deleted. In addition to the teacher's ability to delete the content, the administrator will also check for leaks and delete some inappropriate messages, thereby improving the effectiveness of the information content. Otherwise, the administrator will also summarize the information content during work, thereby improving the reliability of the information content.

\subsection{Teaching Evaluation Module}

Under the conditions of the application of the Web platform, we also need to establish a teaching evaluation system to analyze the teaching effect of teachers and the learning situation of students. Judging from the current application situation, we can use the April algorithm to process it to obtain more objective application data [9]. Using data to compare the results can help teachers understand the learning situation of students on the content of the course and clarify some problems existing in the current stage of learning. In this way, teachers can formulate corresponding measures to solve them, so as to continuously optimize the curriculum teaching system. This evaluation system can also promote the continuous optimization of students' mental health level under effective teaching methods, thereby enhancing the application effect of the platform system.

\subsection{Database Module Design}

There are certain differences in the psychological quality of students themselves, as well as different ways of thinking. In order to meet the diversified development requirements of students, we should rely on the Web platform to design database modules to accumulate data, so as to establish a reliable data system for the application of the mental health teaching system. In the design process of the actual database module, we need to separately establish the data application structure used by students, teachers and administrators. We are supposed to integrate and update the data information content according to actual application requirements simultaneously, so as to improve the application value of the information content and improve the application effect of the information itself [10].

\subsection{Interactive Platform Module Design}

The main purpose of establishing a mental health education system is to help students establish a sound value concept, so that students have a strong ability to distinguish when dealing with things. And in practical applications, the design of interactive platform modules is also one of the most important content. The role of the interactive platform is to meet the smooth completion of information interaction between teachers and students and to effectively manage existing platform resources. Furthermore, the interactive platform also increases the interaction between teachers and students, which is conducive to solving some problems in time and speeding up the improvement of students' comprehensive ability [11].

\subsection{Resource Sharing Platform Design}

In addition to the system content mentioned earlier, in the design of the mental health education system, we also need to do a good job in the design of the resource sharing platform. In the specific design, first of all, we need to separately establish a data resource sharing structure for students and teachers. Secondly, we need to integrate data and information content according to actual application requirements, so that it can provide users with convenient conditions in a targeted manner, thereby optimizing resource information [12].

\section{Conclusion}

In summary, in the context of increasing emphasis on mental health education, higher vocational colleges have a long way to go, and the construction of their system is a long-term project. With the help of the application advantages of the Web platform itself, the establishment of the education system can make it fit the current development of higher vocational colleges, which has a positive effect on the improvement of students' mental health.

\section{References}

1. Liu Liqin. The construction of the mental health education curriculum system in public security colleges-from the perspective of Mozi's vocational education thought in traditional culture[J]. Journal of Guangxi Police College, 2020, 33(02): 108-112.

2. Wang Shuchi. A preliminary study on the construction of the "Trinity" management system of mental health education in higher vocational colleges [J]. Hubei Agricultural Mechanization, 2020(07): 48.

3. Yu Jing. Research on the Construction of Curriculumbased Teaching Circle Mode in Higher Vocational Colleges__ Taking the Course of "College Students' Mental Health Education" as an example [J]. Journal of Zhejiang Business Vocational and Technical College, 2020, 19(01): 51-54 .

4. Fu Xiaochun, Liu Juan, Ouyang Yangfan. Construction and Practice of Entrepreneurship Education System in Health Vocational Colleges-_ Based on Timmons' Entrepreneurship Education Theory $[\mathrm{J}]$. Guangdong Vocational and Technical Education and Research, 2019(06):68-70. 
5. Yi Ying. On the construction of the "Internet +" mental health education model in higher vocational colleges[J]. Journal of Liaoning Teachers College (Social Science Edition), 2019(06): 79-80.

6. Gao Yanli. Discussion on the construction of mental health education model for art students in private higher vocational colleges from the perspective of positive psychology[J]. Modern Economic Information, 2019(19): 368-369+371.

7. Lu Xuan. Research on the Construction of Mental Health Curriculum System in Art Vocational Colleges from the Perspective of Psychological Capital[J]. Cultural Innovation Comparative Research, 2019, 3(22): 129-130.

8. He Xingmei. The construction of the mental health education model for college students in higher vocational colleges under the background of "Internet +"[J]. Southern Agricultural Machinery, 2019, 50(12): $103+109$.

9. Ren Yongyou, Cui Shujun, Xing Fengmin, Wang Zhenyu. Research on the Construction of Mental Health Education System for College Students in Higher Vocational Colleges[J]. Journal of State Grid Institute of Technology, 2019, 22(03): 74-77.

10. Chen Qin, Kong Jin. Research on the Construction of Mental Health Education Model for College Students in Higher Vocational Colleges Based on Positive Psychology [J]. Journal of Chuzhou Vocational and Technical College, 2019, 18(02): 19-23.

11. Jiang Shaojun. The construction of the mental health education model for freshmen in higher vocational colleges from the perspective of psychology[J]. Science and Technology Information, 2019, 17(15): $170+182$

12. Wang Yu. Construction of the Curriculum System of Mental Health Education for Students in Art Higher Vocational Colleges [J]. Journal of Guilin Teachers College, 2019, 33(03): 128-131. 